\title{
A Cephalometric Analysis for Evaluation of Changes in Soft Tissues in the Regions of the Upper and Lower Lips and Chin due to Orthognathic Maxillary Advancement Surgery
}

\author{
Hélcio Tadeu Ribeiro ${ }^{1}$ Ana Célia Faria ${ }^{1}$ Alexandre Laguna Terreri ${ }^{1}$ Francisco Veríssimo de Mello-Filho ${ }^{1}$ \\ ${ }^{1}$ Department of Ophthalmology, Otorhinolaryngology and Head and \\ Neck Surgery, Faculty of Medicine of Ribeirão Preto, Universidade de \\ São Paulo, Ribeirão Preto, SP, Brazil \\ Int Arch Otorhinolaryngol 2014;18:57-62.

\begin{abstract}
Address for correspondence Ana Célia Faria, PhD, Department of Ophthalmology, Otorhinolaryngology and Head and Neck Surgery Bandeirantes, 3900-Ribeirão Preto, SP, Brazil 14049-900
\end{abstract} \\ Faculty of Medicine of Ribeirão Preto, Universidade de São Paulo Av. \\ (e-mail: anacfaria@usp.br).
}

\begin{abstract}
Keywords

- orthognathic surgery

- cephalometry

- maxilla

Introduction There is currently no consensus regarding the best method for predicting the changes in soft tissues due to the modification of hard tissues in orthognathic surgery.

Objective To measure the changes in soft tissues of the upper lip, lower lip, and chin regions due to the modifications of hard tissues caused by orthognathic maxillary advancement surgery using a cephalometric methodology.

Methods The study was conducted on 35 patients with dentoskeletal and facial deformities submitted to orthognathic maxillary advancement surgery. Two teleradiographs were taken: one during the preoperative period and the other 1 year after the surgery, on which the cephalometric tracing was drawn.

Results A strong correlation $(r=0.747)$ was demonstrated in the horizontal analysis between the hard $A(A h)$ point (located in the deepest point of the anterior curvature of the maxilla) and the soft $A(A s)$ point in the advancement of the maxilla, with a mean variation of $0.859 \%$ occurring in As with each $1 \%$ variation of the Ah point. A mean variation of $0.698 \%$ occurred in the superior soft prostion point (prolongation of the superior hard prostion point to its corresponding point on soft tissue) for each $1 \%$ variation in the superior hard prostion point (bone point located at the junction of the alveolar process with the crown of the upper incisors).

Conclusion The cephalometric methodology applied here revealed that the soft tissues of the upper lip accompanied 70 to $80 \%$ of the movement of hard tissues in maxillary advancement and that the soft tissues of the lower lip did not change or showed no significant changes.
\end{abstract}

\section{Introduction}

Cephalometry as used today took several years to be refined and went through various phases for the establishment of its methodology. In the Renaissance, more elaborate techniques arose for the measurement of the body and the face. Albrecht Durer (1417-1518) and Leonardo da Vinci (1452-1519) drew human faces on which they traced straight lines between different points joining homologous anatomical structures that divided the head with vertical and horizontal lines. received

July 22, 2013

accepted

September 10, 2013
DOI http://dx.doi.org/

10.1055/s-0033-1361082. ISSN 1809-9777.
Copyright ( $\odot 2014$ by Thieme Publicações License terms Ltda, Rio de Janeiro, Brazil

() (1) $\odot \circledast$ 
Variations in the direction of these lines were considered to be deviations of facial structures. These were probably the first evaluations of facial changes or asymmetries. Curiously, both artists used the true vertical line (TVL; plumb line) and the natural head position (NHP) as reference bases. ${ }^{1}$

The manual method has been long used to execute the cephalometric tracing and to obtain the angular and linear measurements necessary for its interpretation. As the study of cephalometry progressed, new measurements were presented by different investigators and the amount of information added to the cephalograms became quite extensive.

Legan and Burstone proposed a cephalometric analysis of soft tissues directed at orthosurgical patients to complement previously published analyses of hard tissues. ${ }^{2,3}$ The soft tissues that cover the teeth and bones are highly variable in thickness, and the measurements of hard tissues may diverge from the facial contour of soft tissues presented by the patient. The use of arbitrary reference planes in skeletal analysis, such as the Frankfurt horizontal plane and the sella-nasion, to evaluate the maxillary and mandibular positioning may also lead to some imprecision because these reference planes may present variations. The main objective of orthosurgical treatment is to improve the appearance of the face rather than to cause the cephalometric measurements of the patients to be within normal patterns. Although in many cases the improvement of facial appearance causes the cephalometric measurements to be close to normal, in some cases this may not be true.

A study published in 1999 described a new way to evaluate the lateral cephalometric radiographies using the TVL. ${ }^{4}$ This method was denoted "soft tissue cephalometric analysis," whereby the horizontal and vertical positions of the reference points of soft tissues are evaluated in relation to the TVL.

There is currently no consensus regarding the best method for predicting the changes in soft tissues due to the modification of hard tissues in orthognathic surgery.

The objective of the present study was to measure the changes in the soft tissues of the upper lip, lower lip, and chin regions resulting from orthognathic maxillary advancement surgery using a cephalometric methodology.

\section{Methods}

\section{Population}

The study was conducted on 35 Caucasian patients of both sexes age 18 to 50 years, with dentoskeletal facial deformities. The research protocol was approved by the Ethics Committee of Institution (protocol no. 6849/2007) and all patients gave informed consent to the work.

The inclusion criteria were: adult patients with class III skeletal facial pattern; absence of cleft lip/palate or any other developmental anomaly; patients submitted only to maxillary advancement by the Le Fort I technique with no vertical modification or other complementary procedures.

Exclusion criteria were: patients whose surgery generated vertical maxillary movement of more than $3 \mathrm{~mm}$; patients who required some type of graft or interpositional bone substitute during surgery; impossibility of superposition of structures of the skull base on the pre- and postoperative cephalometric radiographies; patients submitted to any type of mentoplasty so that there would be no additional factor that might alter the movement of the lower lip; patients who did not accept to participate in the study or did not follow the preestablished protocol.

\section{Lateral Cephalometric Radiographies}

Lateral cephalometric radiographies were obtained from each patient by the same operator. All patients were instructed to assume NHP using as reference a mirror positioned in front of them. The following protocol was adopted to obtain NHP: erect position, with the feet $\sim 10 \mathrm{~cm}$ apart; gaze fixed on the reflected image of one's eyes using the mirror in front; the auricular olives, when inserted in the tragus, should maintain a slight contact with the skin to prevent possible elevations of the neck or the head. The nasion positioner was adapted in a gentle manner so that the median sagittal plane would remain perpendicular to the horizontal plane. TVL was obtained using a metal wire attached to a plumb line positioned close to the anterior margin of the film holder frame, so that it would appear in front of the soft tissue profile of the patient.

Two teleradiographs were taken for each patient, one of them preoperatively and the other after the orthognathic surgery.

\section{Cephalometry}

The cephalometric tracings were obtained by a manual method using a $0.5-\mathrm{mm}$ pencil, a millimeter ruler, and acetate paper laterally attached with adhesive tape to the cephalometric radiographies, which were transilluminated in a table negatoscope for better identification of the anatomical structures. The tracings were performed in a dark room for better visualization of the radiographies. Points and lines were marked for the determination of angles and lateral measures of hard and soft tissues.

Two reference lines were first traced on the pre- and postoperative radiographies: a horizontal axis (HA) line and a vertical axis (VA) line. The HA line was defined as the one traced 12 degrees below the Base of the sella-Nasion line starting from the base of the sella (-Fig. 1). This reference line traced in this manner becomes more parallel to the natural horizontal plane. The VA line was defined as the line perpendicular to the HA line passing through the base of the sella (-Fig. 1).

Points located on hard and soft tissues were established for the execution of the measurements. The points located on the hard tissues were first marked. The points on soft tissues that composed the horizontal lines were traced starting from VA, passing through the cephalometric point on hard tissue and continuing to the limit of the corresponding soft tissue (-Fig. 2).

\section{Cephalometric Analysis}

The following cephalometric points were marked (-Fig. 2):

BST (base of the sella turcica): most inferior point of the sella turcica that intersects the oblique line starting from $\mathrm{N}$ 


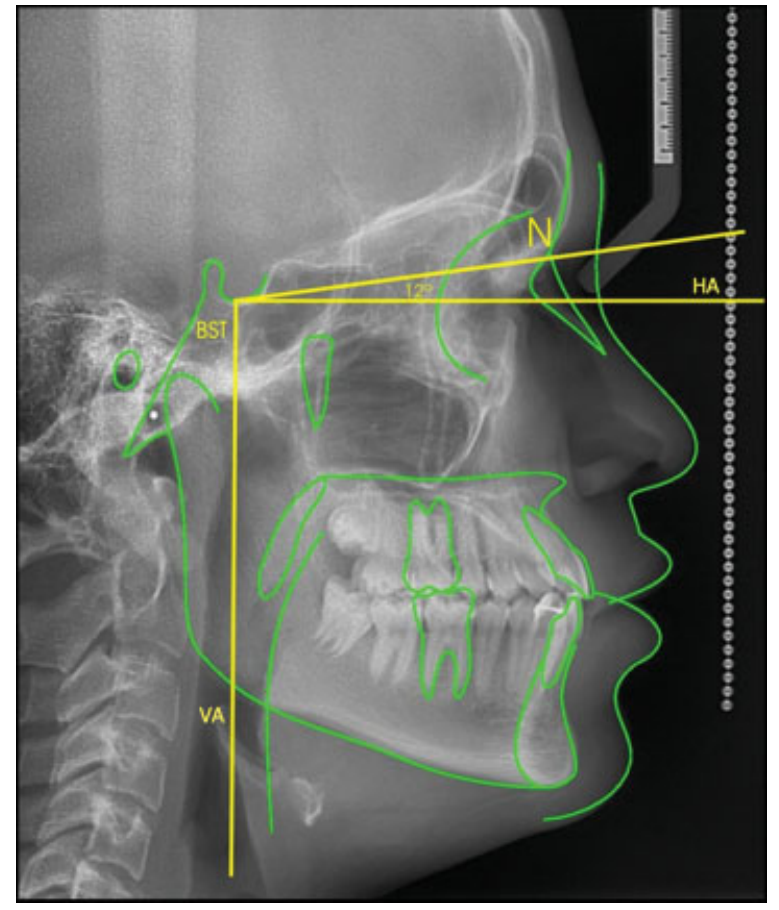

Fig. 1 Horizontal axis obtained with 12 degrees below the BST-N line and vertical axis perpendicular to the horizontal axis.

Abbreviations: BST, base of the sella turcica; HA, horizontal axis; N, nasion point; $V A$, vertical axis.

$\mathrm{N}$ (nasion point): most anterior point of the frontonasal suture where the lines of the glabella profile meet those of the nasal bones

Ah (hard A point): the deepest point of the anterior curvature of the maxilla between the anterior nasal spine and the alveolar process

As (soft A point): prolongation of point A to its corresponding point on soft tissue

PrSh (superior hard prostion point): point located on the junction of the alveolar process with the crown of the upper incisors

PrSs (superior soft prostion point): prolongation of the PrSh to its corresponding point on soft tissue

PrIh (inferior hard prostion point): point located on the junction of the alveolar process and the crown of the lower incisors

PrIs (inferior soft prostion point): point on soft tissue corresponding to the prolongation of PrIh to its corresponding point on soft tissue

Bh (hard B point): deepest point of the anterior concavity of the mandible

Bs (soft B point): point on soft tissue corresponding to the prolongation of point $B$ to its corresponding point on soft tissue

Pogh (hard pogonion point): most anterior point of the contour of the mandibular symphysis

Pogs (soft pogonion point): point on soft tissue corresponding to the prolongation of the Pog point to its corresponding point on soft tissue

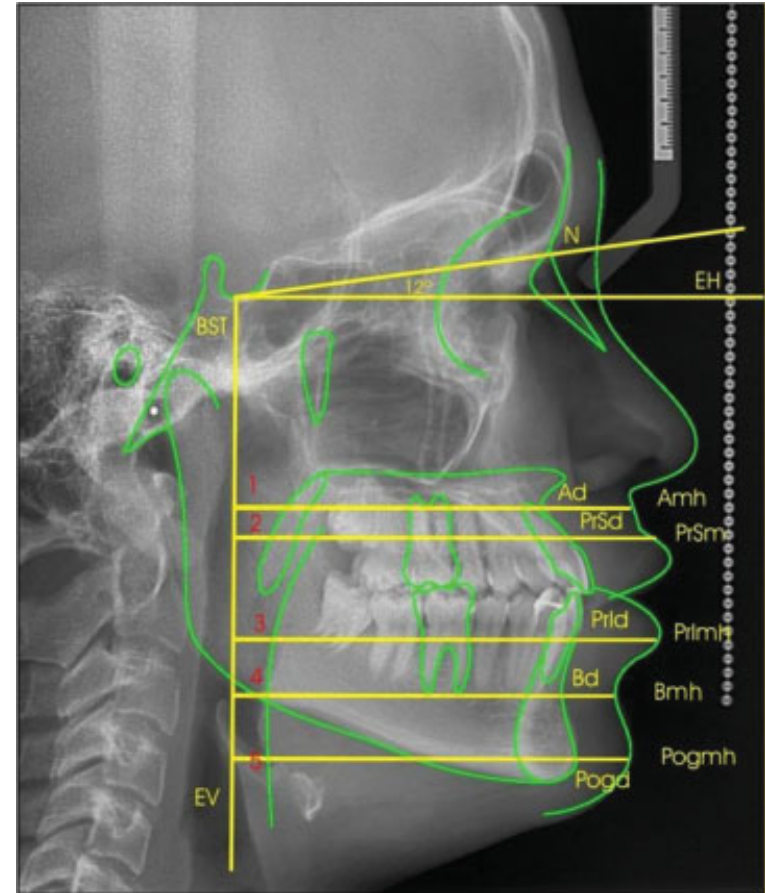

Fig. 2 Cephalometric points used in the present study. BST (base of the sella turcica): most inferior point of the sella turcica that intersects the oblique line starting from $\mathrm{N} ; \mathrm{N}$ (nasion point): most anterior bone point of the frontonasal suture; Ah (hard A point): bone point located in the deepest point of the anterior curvature of the maxilla between the anterior nasal spine and the alveolar process; As (soft A point): prolongation of point $A$ to its corresponding point on soft tissue; PrSh (superior hard prostion point): bone point located at the junction of the alveolar process with the crown of the upper incisors; PrSs (superior soft prostion point): prolongation of the PrSh to its corresponding point on soft tissue; Prlh (inferior hard prostion point): point located at the junction of the alveolar process with the crown of the lower incisors; Prls (inferior soft prostion point): point on soft tissue corresponding to the prolongation of Prlh to its corresponding point on soft tissue; Bh (hard B point): deepest point of the anterior concavity of the mandible; Bs (soft B point):point on soft tissue corresponding to the prolongation of point $B$ to its corresponding point on soft tissue; Pogh (hard pogonion point): most anterior point of the contour of the mandibular symphysis; Pogs (soft pogonion point): point on soft tissue corresponding to the prolongation of the Pog point to its corresponding point on soft tissue. Horizontal lines: BST-N: trace from point BST to point N; Line 1: line that starts perpendicular to the vertical axis, passing through Ah and continuing to As; Line 2: line that starts perpendicular to the vertical axis, passing through PrSh and continuing to PrSs; Line 3: line that starts perpendicular to the vertical axis, passing through Prlh and continuing to Prls; Line 4: line that starts perpendicular to the vertical axis, passing through $\mathrm{Bh}$ and continuing to $\mathrm{Bs}$; Line 5 : line that starts perpendicular to the vertical axis, passing through Pogh and continuing to Pogs.

\section{Horizontal Lines}

The following horizontal lines were marked:

BST-N: line traced from the BST point to the $\mathrm{N}$ point

Line 1: line starting perpendicular to the vertical axis, passing through $\mathrm{Ah}$ and continuing to As

Line 2: line PrSh and continuing to PrSs

Line 3: line starting perpendicular to the vertical axis, passing through PrIh and continuing to PrIs 
Line 4: line starting perpendicular to the vertical axis, passing through $\mathrm{Bh}$ and continuing to $\mathrm{Bs}$

Line 5: line starting perpendicular to the vertical axis, passing through Pogh and continuing to Pogs

Le Fort I osteotomy was the surgical technique used for maxillary advancement.

\section{Statistical Analysis}

The Pearson correlation coefficient was used to determine the relationship between the changes in soft tissue and those of bone tissue. The calculation was used to test the hypothesis of a direct relationship between movement of bone tissue and points on soft tissue, yielding a correlation between the magnitudes analyzed. We used the Pearson correlation coefficient $(r)$, which determines the variation of the modification of soft tissue in relation to hard tissue with the following meaning: $R$ of 0 to 0.49 , no correlation between the measurements analyzed; $R$ from 0.5 to 1.0 , there is a correlation between hard and soft tissue.

We also used the intercept (I), which is the linear coefficient of the model, Ahj. $R^{2}$ determines the percent variability of the data applied by the linear model coefficient of determination. The level of significance was set at $p<0.05$.

Complex statistical calculations were performed and graphs and dispersal diagrams were generated to visualize the relationship of dislocation at points of bone and soft tissue.

\section{Results}

The results of the present study demonstrated that there was a strong correlation $(r=0.747)$ in the horizontal analysis between $\mathrm{Ah}$ and As in maxillary advancement, with a mean $0.859 \%$ variation in As for each $1 \%$ variation in the Ah point (-Fig. 3).

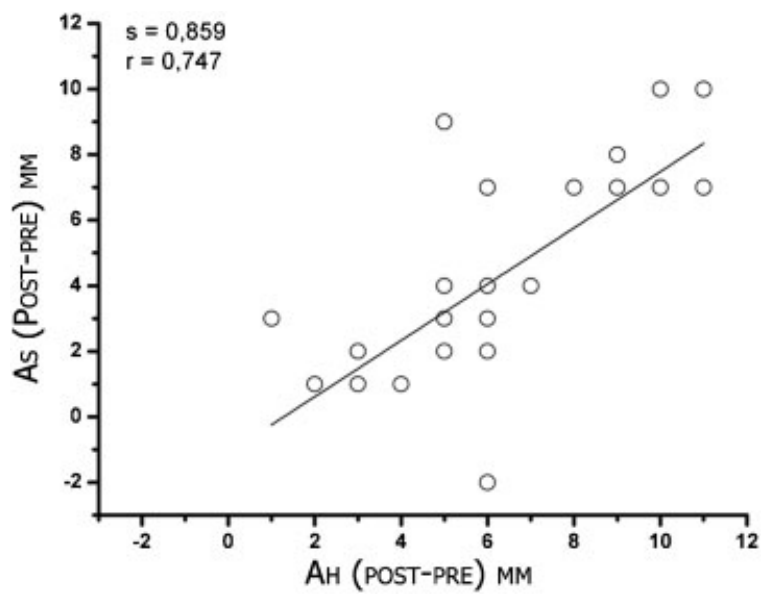

Fig. 3 Horizontal relationship between Ah and As (line 1) in maxillary advancement. It can be seen that a strong correlation $(r=0.747)$ exists between $A h$ and As in the horizontal analysis The graph demonstrates that a mean $0.859 \%$ variation in As occurs for each $1 \%$ variation of the Ah point $\left(y=0.859^{*} x\right)$.

Abbreviations: Ah, hard A point; As, soft A point.
-Fig. 4 demonstrates that a mean 0.698\% variation in PrSs occurred for each $1 \%$ variation in the PrSh point due to maxillary advancement surgery.

Analysis of the effects of maxillary advancement surgery on the soft tissues of the mandible using the horizontal relation of PrIs (line 3), Bs (line 4), and Pogs (line 5) with Ah demonstrated that the three soft points had zero or close to zero median and mean (-Fig. 5).

\section{Discussion}

The result of orthognathic surgeries in terms of facial esthetics depends on the effects of the changes in soft tissues

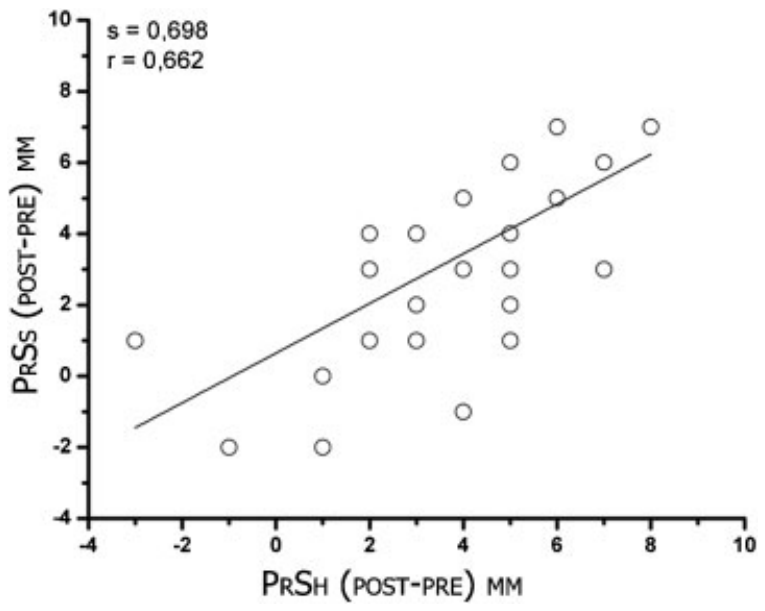

Fig. 4 Horizontal relationship between PrSh and PrSs (Line 2) in maxillary advancement. It can be seen that a strong correlation $(r=0.662)$ exists between PrSh and PrSs in the horizontal analysis. The graph demonstrates that a mean $0.698 \%$ variation in PrSs occurs for each $1 \%$ variation of the PrSh point $\left(y=0.698{ }^{*} x\right)$.

Abbreviations: PrSh, superior hard prostion point; PrSs, superior soft prostion point.

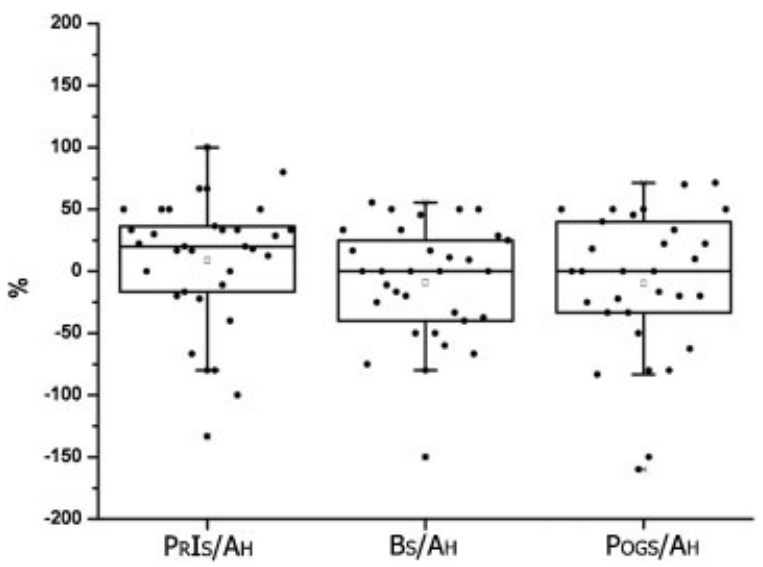

Fig. 5 Horizontal relationship between Prls (line 3), Bs (line 4), and Pogs (line 5) and Ah in maxillary advancement. It can be seen that the three soft points have zero or close to zero mean and median. Abbreviations: Ah, hard A point; Bs, soft B point; Pogs, soft pogonion point; Prls, inferior soft prostion point; PrSh, superior hard prostion point; PrSs, superior soft prostion point. 
that accompany the repositioning of bone structures. Thus, in surgical planning it is extremely important to know the correct relation between bone movements and their effects on soft tissues. The more precise this information, the greater the surgeon's safety in planning the orthognathic surgery, not only for functional correction but also for the best esthetic result, so strongly desired by all patients. The identification of the aesthetic factors and the prediction of the final profile of the facial soft tissues play important roles in planning the orthognathic treatment. ${ }^{5}$ However, it can be seen that the literature is quite divergent regarding the quantitation of the changes in soft tissues due to the bone movements performed in orthognathic surgeries.

In the present study, the evaluation of maxillary advancement surgery revealed that the amount of bone advancement of point Ah is reflected in $86 \%$ advancement of point As (-Fig. 3). This means that for each 1-mm advancement of the maxilla, the soft tissue of the upper lip in the subnasal region will advance by $0.86 \mathrm{~mm}$. This movement shows a strong correlation between hard tissue and changes in soft tissue $(r=0.75)$. A survey of the literature showed that several authors who evaluated the changes in soft tissues due to maxillary advancement in point A detected 60 to $83 \%$ variation in such changes in the various studies. ${ }^{6-9}$ In view of such variation in results, it is difficult to define the result that best reflects the reality. The findings of the present study were close to those reported by Brooks et al. ${ }^{10} \mathrm{~A}$ possible explanation for such variability is the methodology used in each study, as well as variations in race, amplitude of movements, or even sex of the patients studied. Some studies have used a distinct methodology based on angular measurements. In the present study, we used linear measurements considered to be more appropriate and applied a horizontal line with a well-defined bone point to find its corresponding soft point. Tissue thickness was measured with considerable precision at the same soft and hard points before and after surgery. Thus we believe that we can safely state that, in maxillary advancement, the Ah point produces on average an $86 \%$ advancement of its corresponding soft tissue. We consider this finding to be important because maxillary movements are frequently used in daily clinical practice and, depending on the dimension of this movement, it is possible to predict the position of the upper lip. Thus the reestablishment of a harmonic profile can be predicted, as well as an appropriate upper lip-lower lip relationship with a better final esthetic result.

In the region of the upper lip vermilion, PrSs point, in maxillary advancement surgery, we observed that the horizontal advancement of soft tissue was $0.70 \mathrm{~mm}$ for each $1 \mathrm{~mm}$ of bone advancement of the PrSh point (i.e., 70\% of the amount of bone advancement; - Fig. 4). This means that the upper lip in the vermilion region has a 30\% smaller thickness after surgery, with a strong correlation between bone movement and changes in soft tissue $(r=0.662)$. Other studies about the modification of this point due to maxillary advancement have reported values ranging from 50 to $75 \%{ }^{6,11-15}$ The results of the present study agree with those reported by Arnett et al, ${ }^{16}$ but in any case, there is general agreement that the upper lip does not accompany the magnitude of bone movement. Another interesting aspect is that although the soft tissues of the As point advance by $86 \%$, the PrSs point advances by $70 \%$. On this basis, advancement of the upper lip does not occur in a homogeneous manner, with its greater amplitude being in its portion close to the base of the nose, being progressively reduced up to the vermilion by $\sim 16 \%$.

Skeletal maneuvers not only can change the soft tissue morphology in the immediate vicinity, but also that in distant regions owing to the $3 \mathrm{D}$ composite anatomy of the craniofacial region. These changes affect the overall esthetic balance of the face. Thus, maxillofacial surgeons must pay due attention to alterations in the facial form before performing orthognathic surgery. ${ }^{17}$

Does the maxillary advancement surgery provoke changes also in soft tissues of the lower lip region?

Kim et al have confirmed that conventional Le Fort I osteotomy affected areas remote from the osteotomy line. ${ }^{18}$ However, the effect was not very pronounced compared with the nearer areas.

In the present study, analysis of the horizontal changes at points PrIs, Bs, and Pogs (-Fig. 5) in maxillary advancement surgery revealed that, on average or as the median, the thickness of the soft tissues did not change. However, a $25 \%$ variation of more or less may occur in PrIs, Bs, and Pogs. A possible explanation for such variation is that when the maxilla is advanced, the mandible is repositioned in class I occlusion, possibly producing small clockwise or counterclockwise movements. These movements may provoke adjustments of soft tissues in the lower lip and chin region. We noted that the tissues underwent an equal and discrete adjustment of $\sim 25 \%$ or suffered no change. Greater variability occurred in the region of the mentolabial sulcus, possibly causing a slight modification in the shape of the sulcus, turning it less markedly. This type of analysis and the findings obtained here were not detected in the literature.

\section{Conclusion}

Based on the methodology employed and the results obtained, the soft tissues of the upper lip, lower lip, and chin regions were found to have different responses depending on the osseous movement applied to the maxilla. In general, maxillary advancement produces an $80 \%$ projection of the upper lip and a discrete change or no change in the tissues of the lower lip and chin.

\section{References}

1 Finlay LM. Craniometry and cephalometry: a history prior to the advent of radiography. Angle Orthod 1980;50(4):312-321

2 Legan HL, Burstone CJ. Soft tissue cephalometric analysis for orthognathic surgery. J Oral Surg 1980;38(10):744-751

3 Burstone CJ, James RB, Legan H, Murphy GA, Norton LA. Cephalometrics for orthognathic surgery. J Oral Surg 1978;36(4): 269-277 
4 Arnett GW, Jelic JS, Kim J, et al. Soft tissue cephalometric analysis: diagnosis and treatment planning of dentofacial deformity. Am J Orthod Dentofacial Orthop 1999;116(3):239-253

5 Aydil B, Özer N, Marşan G. Facial soft tissue changes after maxillary impaction and mandibular advancement in high angle class II cases. Int J Med Sci 2012;9(4):316-321

6 Lines PA, Steinhauser EW. Soft tissue changes in relationship to movement of hard structures in ortognathic surgery: a preliminary report. J Oral Surg 1974;32:891-896

7 Carlotti AE Jr, Aschaffenburg PH, Schendel SA. Facial changes associated with surgical advancement of the lip and maxilla. J Oral Maxillofac Surg 1986;44(8):593-596

8 Medeiros PJ, Quintão CCA, Menezes LM. Avaliação da estabilidade do perfil facial após tratamento cirúrgico. Ortodontia Gaúcha 1999;3:5-23

9 Vigorito JW. Traçado predictivo: proposta de um método em caso de maloclusões dentárias com indicações para cirurgia ortognática. Ortodontia 2000;33:63-73

10 Brooks BW, Buschang PH, Bates JD, Adams TB, English JD. Predicting upper lip response to 4-piece maxillary LeFort I osteotomy. Am J Orthod Dentofacial Orthop 2001;120(2):124-133

11 Mansour S, Burstone C, Legan H. An evaluation of soft-tissue changes resulting from Le Fort I maxillary surgery. Am J Orthod 1983;84(1):37-47
12 Epker B, Stella JP, Fish L. Dentofacial Deformities: An Integrated Orthodontic Surgical Approach. St. Louis, MO: Mosby; 1985: $3-70$

13 Hernandez-Orsini R, Jacobson A, Sarver DM, Bartolucci A. Shortterm and long-term soft tissue profile changes after mandibular advancements using rigid fixation techniques. Int J Adult Orthod Orthognath Surg 1989;4:209-218

14 Jensen AC, Sinclair PM, Wolford LM. Soft tissue changes associated with double jaw surgery. Am J Orthod Dentofacial Orthop 1992; 101(3):266-275

15 Gregoret J. Ortodontia e cirurgia ortognática, diagnóstico e planejamento. Barcelona, Spain: Livraria Santos; 1999

16 Arnett GW, McLaughlin RP. Planejamento facial e dentário para ortodontistas e cirurgiões bucomaxilofaciais. São Paulo, Brazil: Artes Médicas; 2004

17 Vasudavan S, Jayaratne YSN, Padwa BL. Nasolabial soft tissue changes after Le Fort I advancement. J Oral Maxillofac Surg 2012;70(4):e270-e277

18 Kim YI, Park SB, Son WS, Hwang DS. Midfacial soft-tissue changes after advancement of maxilla with Le Fort I osteotomy and mandibular setback surgery: comparison of conventional and high Le Fort I osteotomies by superimposition of cone-beam computed tomography volumes. J Oral Maxillofac Surg 2011; 69(6):e225-e233 